\title{
On computing the semi-sum of two integers
}

\author{
Salvatore Ruggieri \\ Dipartimento di Informatica, Università di Pisa, \\ Via F. Buonarroti 2, 56125 Pisa ITALY \\ ruggieri@di.unipi.it
}

\begin{abstract}
We derive a sound program for computing the semi-sum of two integers using only integer operators and without incurring overflow.
\end{abstract}

Keywords: formal methods, program derivation.

\section{Problem statement}

Given two integers $a$ and $b$, we wish to compute ${ }^{1}\lfloor(a+b) / 2\rfloor$, also called the semisum of $a$ and $b$. While the problem may seem elementary, we must tackle some implementation issues that demand a non-trivial solution.

Consider the "first-answer" solution consisting of the simple C/Java expression $(\mathrm{a}+\mathrm{b}) / 2$. First, recall that division between integer expressions in C, Java and other common languages, rounds towards zero. Specifically, for an integer $\mathrm{n}$, the expression $\mathrm{n} / 2$ evaluates to $\lfloor n / 2\rfloor$ when $n \geq 0$ and evaluates to $\lceil n / 2\rceil$ when $n \leq 0$. Therefore, $(\mathrm{a}+\mathrm{b}) / 2$ is a sound implementation of $\lfloor(a+b) / 2\rfloor$ only when $(a+b) \geq 0$.

Second, computation of sub-expression $(a+b)$ may cause an overflow, i.e. its value may be out of the range of representable integers. Let us write $\operatorname{rep}(n)$ iff $n$ is a representable integer. For now, we do not consider any specific representation (later on we will assume a two's complement representation) but we only assume that $\operatorname{rep}(a) \wedge \operatorname{rep}(b)$, that $\operatorname{rep}(0)$ and that:

$$
\forall m, n, p \in Z . \quad(\operatorname{rep}(m) \wedge \operatorname{rep}(n) \wedge m \leq p \leq n \quad \Rightarrow \quad \operatorname{rep}(p)) .
$$

Since $\min \{a, b\} \leq\lfloor(a+b) / 2\rfloor \leq \max \{a, b\}$, by (1) the semi-sum of $a$ and $b$ is a representable integer.

We are now in a position to formally state the problem specification.

Problem statement. Derive a $C$ program SEMI-SUM such that for $\mathrm{a}, \mathrm{b}, \mathrm{s}$ variables of type int the following Hoare triple is valid:

$$
\{\text { true }\} \text { SEMI-SUM }\{s=\lfloor(a+b) / 2\rfloor\}
$$

and such that all expressions in SEMI-SUM denote representable integers only.

\footnotetext{
${ }^{1}$ Throughout this paper we adopt standard mathematical notation (see e.g. [GKP89]) concerning the floor $(\lfloor\rfloor)$ and ceiling $(\lceil\rceil)$ operators.
} 
Such a calculation occurs quite often in computer programs, e.g. in the wellknown binary search algorithm. As another example, the semi-sum is computed in the C4.5 decision tree induction algorithm [Qui93], where the following (here, simplified) recursive procedure is adopted. Given an array of distinct integers, two elements $a$ and $b$ in the array are selected according to some criterion and then the array is split into two parts: those elements at most the semi-sum of $a$ and $b$, and those elements greater than such a semi-sum. The procedure is recursively applied to each of the two arrays unless their length is less than two.

In testing an implementation of the C4.5 algorithm [Rug02], infinite loops originated from the "first-answer" calculation. First, the computed semi-sum of -3 and -2 was -2 (wrong, since it is -3 ). This led to splitting an array such as $[-3,-2]$ into $[-3,-2]$ and an empty one: recursion on the first split yielded the infinite loop. Second, the computed semi-sum of $2^{30}$ and $2^{30}$ was $-2^{30}$ (wrong, since it is $\left.2^{30}\right)$. This led to splitting an array such as $\left[2^{30}, 2^{30}\right]$ into itself and an empty one: as before, this led to an infinite loop.

\section{Second-answer calculation}

As discussed in the previous section, the "first-answer" calculation is a sound implementation only when $(a+b) \geq 0$ and $\operatorname{rep}(a+b)$, i.e.:

$$
\{(a+b) \geq 0 \wedge \operatorname{rep}(a+b)\} \mathrm{s}=(\mathrm{a}+\mathrm{b}) / 2 ;\{s=\lfloor(a+b) / 2\rfloor\} .
$$

In $(2)$, the expression $(\mathrm{a}+\mathrm{b})$ denotes a representable integer by assumption, and the expression $(a+b) / 2$ denotes a representable integer since it coincides with the semi-sum of $a$ and $b$, which is representable.

Consider now the case $(a+b) \leq 0$. Using the identity ([GKP89, (3.17)]):

$$
\forall m \in Z . \quad(m=\lfloor m / 2\rfloor+\lceil m / 2\rceil)
$$

we derive: $\lfloor(a+b) / 2\rfloor=(a+b)-\lceil(a+b) / 2\rceil$. Since $(a+b) \leq 0,\lceil(a+b) / 2\rceil$ coincides now with $(\mathrm{a}+\mathrm{b}) / 2$. Therefore:

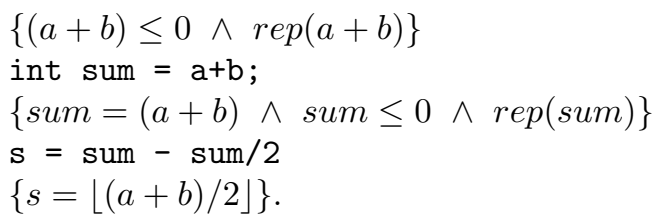

Also, note that sum $\leq\lceil$ sum $/ 2\rceil \leq 0 \wedge \operatorname{rep}($ sum $)$ and $\operatorname{rep}(0)$ imply by (1) $\operatorname{rep}(\lceil\operatorname{sum} / 2\rceil)$, i.e. sum/2 denotes a representable integer. Also $\operatorname{rep}(\operatorname{sum}-\lceil\operatorname{sum} / 2\rceil)$ holds since sum - $\lceil$ sum $/ 2\rceil$ is the semi-sum of $a$ and $b$. Therefore, all expressions denote representable integers. Finally, merging (2) with the last program to get a "second-answer" program SEMI-SA:

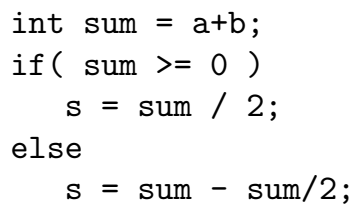

for which the following Hoare triple is valid:

$$
\{r e p(a+b)\} \text { SEMI-SA }\{s=\lfloor(a+b) / 2\rfloor\} .
$$




\section{Nonnegative-division calculation}

There is a second identity ([GKP89, (3.6)]):

$$
\forall x \in R \forall m \in Z . \quad(\lfloor m+x\rfloor=m+\lfloor x\rfloor)
$$

that allows us to rewrite $\lfloor(a+b) / 2\rfloor=\lfloor a+(b-a) / 2\rfloor=a+\lfloor(b-a) / 2\rfloor$. When $a \leq b$, the value $b-a$ is a non-negative integer, and $\lfloor(b-a) / 2\rfloor$ coincides with $(b-a) / 2$. Therefore:

$$
\{a \leq b \wedge \operatorname{rep}(b-a)\} \mathrm{s}=\mathrm{a}+(\mathrm{b}-\mathrm{a}) / 2 ;\{s=\lfloor(a+b) / 2\rfloor\} .
$$

As in the last section, it is readily checked that $(b-a) / 2$ and $a+(b-a) / 2$ denote representable integers. Similarly, when $b \leq a$ :

$$
\{b \leq a \wedge \operatorname{rep}(a-b)\} \mathrm{s}=\mathrm{b}+(\mathrm{a}-\mathrm{b}) / 2 ;\{s=\lfloor(a+b) / 2\rfloor\} .
$$

We can then conclude that for the program SEMI-NND:

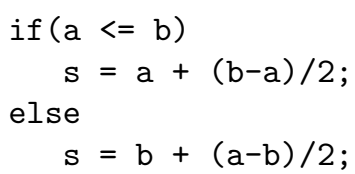

the following Hoare triple is valid $^{2}$ :

$$
\{\operatorname{rep}(\max \{a, b\}-\min \{a, b\})\} \text { SEMI-NND }\{s=\lfloor(a+b) / 2\rfloor\} .
$$

\section{Semi-sum calculation}

Both SEMI-SA and SEMI-NND make a precondition on sub-expressions in order to prevent overflow. A way to satisfy those preconditions is to cast $a$ and $b$ up to a larger numeric data type (e.g., from 32-bit to 64-bit integers), and then to cast the result back to the original data type. However, it may be the case that a larger data type is not available. In this section, we derive a general solution.

Consider again the triple (3). It differs from the problem specification in making the additional assumption $\operatorname{rep}(a+b)$. We observe:

$$
\begin{aligned}
\operatorname{rep}(a+b) \Leftarrow & \{(1) \text { with } p=(a+b), m=a \text { and } n=b\} \\
& \operatorname{rep}(a) \wedge \operatorname{rep}(b) \wedge a \leq(a+b) \wedge(a+b) \leq b \\
\equiv \quad & \{\operatorname{rep}(a), \operatorname{rep}(b), \text { cancellation }\} \\
& a \leq 0 \leq b
\end{aligned}
$$

Analogously, we derive $\operatorname{rep}(a+b) \Leftarrow b \leq 0 \leq a$. By the consequence rule of Hoare logic, these two implications and (3) lead to:

$$
\{\min \{a, b\} \leq 0 \leq \max \{a, b\}\} \text { SEMI-SA }\{s=\lfloor(a+b) / 2\rfloor\} .
$$

Let us apply the same reasoning to the triple (4). For simplifying the notation, let $x=\min \{a, b\}$ and $y=\max \{a, b\}$. We have:

$$
\begin{aligned}
& \operatorname{rep}(y-x) \Leftarrow \begin{array}{c}
\{(1) \text { with } p=(y-x), m=0 \text { and } n=y\} \\
\operatorname{rep}(0) \wedge \operatorname{rep}(y) \wedge 0 \leq(y-x) \wedge(y-x) \leq y
\end{array} \\
& \equiv \quad \begin{array}{l}
\{\operatorname{rep}(0), \operatorname{rep}(a), \operatorname{rep}(b), x \leq y, \text { cancellation }\} \\
0 \leq x
\end{array}
\end{aligned}
$$

${ }^{2}$ Note that by (1), $0 \leq a \wedge 0 \leq b \Rightarrow \operatorname{rep}(\max \{a, b\}-\min \{a, b\})$. By the consequence rule of Hoare logic, (4) implies $\{0 \leq a \wedge \overline{0} \leq b\}$ SEMI-NND $\{s=\lfloor(a+b) / 2\rfloor\}$, which states that SEMI-NND is sound for computing the semi-sum of two representable natural numbers. 
Also, we can show that $\operatorname{rep}(y-x)$ if $y<0$. In order to achieve this, we assume from now on the standard two's complement representation of integers using $p$ bits plus sign: integers representable with the int data type range then from $-2^{p}$ to $2^{p}-1$. In addition to (1) and to rep(0), two's complement notation implies:

$$
\forall n \in Z . \quad(\operatorname{rep}(n) \wedge n<0 \Rightarrow \operatorname{rep}(-n-1)) .
$$

Let us show now that $\operatorname{rep}(y-x)$ if $y<0$.

$$
\begin{aligned}
\operatorname{rep}(y-x) \Leftarrow & \{(1) \text { with } p=(y-x), m=0 \text { and } n=(-x-1)\} \\
& \quad \operatorname{rep}(0) \wedge \operatorname{rep}(-x-1) \wedge 0 \leq(y-x) \wedge(y-x) \leq(-x-1) \\
\equiv & \quad\{\operatorname{rep}(0), x \leq y, \text { cancellation }\} \\
& \operatorname{rep}(-x-1) \wedge y<0 \\
\Leftarrow & \quad\{(6) \text { with } n=x\} \\
& \quad \operatorname{rep}(x) \wedge x<0 \wedge y<0 \\
\equiv \quad & \{\operatorname{rep}(a), \operatorname{rep}(b), x \leq y\} \\
& y<0
\end{aligned}
$$

By the consequence rule of Hoare logic, the last two implications and (4) lead to:

$$
\{(0 \leq \min \{a, b\} \vee \max \{a, b\}<0)\} \text { SEMI-NND }\{s=\lfloor(a+b) / 2\rfloor\} .
$$

By observing that:

$$
(0 \leq \min \{a, b\} \vee \max \{a, b\}<0) \vee(\min \{a, b\} \leq 0 \leq \max \{a, b\})
$$

we can design our final program SEMI-SUM by combining (5) and (7):

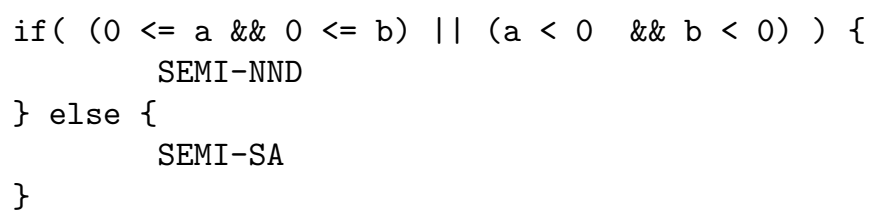

For such a program the specification triple $\{$ true $\}$ SEMI-SUM $\{s=\lfloor(a+b) / 2\rfloor\}$ is valid, and all expressions denote representable integers, i.e. no overflow occurs.

\section{Acknowledgements}

I am grateful to Prof. Roland Backhouse and to Prof. David Gries for their comments on a preliminary version of this paper.

\section{References}

[GKP89] R.L. Graham, D.E. Knuth, and O. Patashnik. Concrete Mathematics: a Foundation for Computer Science. Addison-Wesley Publishing Company, 1989.

[Qui93] J. R. Quinlan. C4.5: Programs for Machine Learning. Morgan Kaufmann, San Mateo, CA, 1993.

[Rug02] S. Ruggieri. Efficient C4.5. IEEE Transactions on Knowledge and Data Engineering, 14:438-444, 2002. 